\title{
Estado mental del adulto mayor usuario dentro del programa líder Juan Luis Londoño de la Cuesta de Cooprosperar en el municipio de Yopal (Casanare).
}

Nidia Milena Moreno López ${ }^{1}$

Ana Patricia Angarita Figueroa ${ }^{2}$

Lizet Lorena Barreto Suarez ${ }^{2}$

Luz Dary Díaz Cárdenas ${ }^{2}$

Patricia Guerra ${ }^{2}$

Claudia Inés Ruiz Moreno ${ }^{2}$

Carlos Alberto Silva Gualdron ${ }^{2}$

María Helena Supelano Valencia ${ }^{2}$

\begin{abstract}
Resumen
El envejecimiento incluye la Agencia y sus manifestaciones son diferentes instituciones en las que la sociedad y el gobierno colombiano brindarán asistencia con el fin de aliviar la realidad del abandono familiar. Este trabajo surge debido se pretende establecer algunas características en las dinámicas interaccionales que se dan entre los habitantes de la tercera edad, como contribución a la comunidad de los adultos mayores, una comprensión sobre sus actitudes, sentimientos, comportamientos y pensamientos.
\end{abstract}

Palabras Clave: estabilidad, emocional, biológico, psicológico, contextual.

1 Investigadora principal, tutora de la Escuela de Ciencias Sociales Artes y Humanidades, de la Universidad Nacional Abierta y a Distancia - CEAD Yopal. Psicóloga, especialista en y Orientación Familiar. Candidata a magister en Gerontología Social, Universidad de Barcelona, nidia.moreno@unad.edu.co, Colombia. 


\title{
Third age adult's mental health as in Cooprosperar's Juan Luis Londoño de la Cuesta leading program in Yopal (Casanare) municipality
}

\begin{abstract}
Aging includes Agency, which is manifested in different institutions where Colombian society and Government will give aging adults support so that their realities, as the abandonment by families, are alleviated. This job arisen from the need to help the older adults community understand their attitudes, feelings, behaviors, thoughts. It intends to set clear some features on the interactional dynamics that occur between the People of the Third Age. United Nations consideration of the very important issue and in economic, cultural, even political and it is implicit The Phenomenon of Change and Adaptation for the Elderly a family and social his Environment.
\end{abstract}

Key Words: stability, emotional, biological, psychological, contextual.

Recibido: 15 de julio 2011

Aceptado: 17 de noviembre 2011

\section{Introducción}

Cuando los adultos entran en una etapa final de la vida, su tarea consiste en contemplar su vida en conjunto y con coherencia. Necesitan aceptar su propia vida tal como la han vivido y creer que lo hicieron lo mejor posible en sus circunstancias (Erikson, 1987), y es en este sentido que se busca establecer el estado mental de los adultos mayores, para así determinar de qué manera se les puede acompañar en este proceso de vida; según lo mencionado por Erikson, repensando a la vejez como un espacio de construcción de sentido.

En este artículo se presenta la indagación obtenida durante un riguroso proceso de observación y aplicación de pruebas, que median el estado cognitivo de los adultos mayores usuarios de los comedores del 20 de Julio, Gabán, Mastranto, la 
Campiña, Hogar Otoño y el comedor del municipio de Maní dentro del programa líder Juan Luis Londoño de la Cuesta de Cooprosperar. Se abordaron aspectos biológicos, psicológicos y contextuales que permitieran la valoración y el análisis general del estado mental de esta población que día a día se ha visto marginada por la sociedad (Monsalve, 2003). Se aplicó el Mini Examen del Estado Mental (MMSE), la escala de Portable Functional Assessment Questionnair (PFEFFER); y para evaluar el estado emocional se aplicó la Escala de Depresión Geriátrica de Yesavage (Abreviada).

Como primer objetivo, se alude a la idea de brindar aquellos elementos conceptuales y metodológicos para la evaluación de las psicopatologías del adulto mayor y del estado cognitivo y afectivo del mismo. En primera instancia se identifican las condiciones relacionadas con el deterioro propio de la edad, pérdida cognitiva, estado emocional y la relación dentro de su contexto.

Se abordan tres dimensiones, la biológica, la psicológica y la emocional. La primera hace referencia al estado de salud, las conductas ligadas a la misma, la condición física, por ejemplo el funcionamiento sensorial y psicomotor, la vista, el oído, el olfato, la fuerza, la coordinación, el tiempo de reacción y los diferentes cambios propios de la edad. En la segunda (psicológica), se pueden evidenciar dimensiones cognitivas, tales como: recordar, orientarse y calcular, teniendo en cuenta que los trastornos cognitivos pueden deberse a diferentes causas, desde trastornos ligeros como el olvido benigno hasta cuadros clínicos graves como la demencia. Se culmina el abordaje con la dimensión emocional: la cual nos muestra los diferentes estados depresivos a los que se ve involucrado el adulto mayor, ya que los síntomas de depresión no son en modo alguno parte necesaria del envejecimiento, siendo así que las personas mayores consideran inútil reportar sus síntomas. Por el contrario, viendo con preocupación la estigmatización social asociada a la enfermedad mental, algunos adultos mayores no mencionan estos problemas por miedo al rechazo o la exclusión social. Para destacar dentro del factor contextual se encuentran las relaciones interpersonales, ya que de ahí se deriva la socialización y relación con sus semejantes, el liderazgo y aquellos conflictos que lo hacen único e irremplazable.

La investigación permitió conocer algunas de las características del adulto mayor, como el de poseer un sentido de institucionalización o apropiación del lugar, y por ende, unas buenas relaciones con sus compañeros. Los roles grupales son fáciles de percibir y son destacables entre compañeros: el líder, los cómicos, los serenos, silenciosos, y los gruñones. Gozan de actividades donde se reúnen con más personas tanto de su misma edad como de diferentes edades, y les da alegría conocer gente nueva. Un punto importante a destacar es la apropiación mental sobre su estado dentro de las etapas del ciclo vital, donde asimilan la llegada de un momento de vida en donde la experiencia adquirida durante toda la vida puede llevar a la realización personal o a la marginación, desesperanza y decepción. 


\section{Metodología}

\section{Tipo de investigación}

Es un estudio descriptivo porque busca identificar y describir sistemáticamente las características de una población dada o de un área de interés, tal como se presenta actualmente y con toda exactitud (Forero, 1998: 83). Esta población son adultos mayores que asisten al programa nacional de alimentación para el adulto mayor Juan Luis Londoño de la Cuesta y Cooprosperar, en total 214 adultos mayores.

\section{Línea de investigación}

Construcción de subjetividades a partir de los procesos de socialización.Esta línea de investigación es con la que se direcciona el trabajo investigativo de la Escuela de Ciencias Sociales Artes y Humanidades de la UNAD, que tiene como objetivo general abordar las dinámicas y problemáticas de orden psicológico desde una perspectiva bio- psico-socio-cultural y comunitaria, contribuyendo al desarrollo de la disciplina y a la construcción de conocimiento desde una perspectiva pluralista e interdisciplinaria que aporte al desarrollo de los individuos y las comunidades, y como objetivos específicos busca indagar los procesos biopsicosociales, culturales y comunitarios que se hacen presentes en las prácticas sociales, así como identificar imaginarios, valores, disposiciones culturales y patrones de organización de contextos que promocionan la conformación de subjetividades y prácticas sociales hacia formas de participación éticas, con conciencia social, como también reconocer los procesos comunitarios y psicosociales que pueden favorecer la acción transformadora de las problemáticas psicosociales, proponer modelos y estrategias para la intervención en problemáticas psicosociales, que favorezcan los procesos participativos autogestionarios y democráticos.

\section{Población objeto de estudio}

Adultos mayores que asisten al programa nacional de alimentación para el adulto mayor Juan Luis Londoño de la Cuesta y Cooprosperar, en total 214 adultos mayores.

\section{Muestra y selección}

Se desarrolló con adultos mayores que asisten al programa de los distintos comedores tales como: Casa hogar otoño, Gabán, Mastranto, 20 de Julio, y el comedor en el municipio de Maní. 


\section{Técnicas e instrumentos de recolección de la información}

Observación directa: Se realizó un riguroso proceso de observación directa en la interacción de los adultos mayores en los comedores a los que asistían, se llevó el registro de la observación en un diario de campo en el cual se plasmaron las observaciones realizadas en las tres dimensiones que se trabajaron; la biológica, la psicológica y la contextual.

Evaluación cognitiva (MMSE) modificada: Este instrumento es útil para el tamizaje de déficit cognitivo, aunque no es un instrumento de diagnóstico. Puede ser administrado por el médico, el enfermero o el trabajador social.

La Escala Pfeffer (Portable Functional Assessment Questionnaire): Instrumento que complementa la evaluación del estado cognitivo con información que es obtenida de un familiar o cuidador del paciente.

La Escala de Depresión Geriátrica de Yesavage (Abreviada): Este instrumento es útil para el tamizaje del estado de depresión así como para guiar al clínico en la valoración del estado de salud mental del individuo. Esta herramienta la puede administrar el médico, el enfermero o el trabajador social.

Entrevista: Se entrevistaron a los adultos mayores con el fin de ampliar la información obtenida en la observación directa y en las pruebas aplicadas.

\section{Resultados}

De acuerdo a las dimensiones analizadas en este estudio se encontró que en la biológica, el $70 \%$ de los adultos entrevistados presentan algunas enfermedades propias de la edad, tales como artritis, hipertensión, problemas con el colesterol, y problemas en los huesos, tensión alta, triglicéridos, diabetes, dado que hacen parte de las enfermedades osteoarticulares, cardiovasculares, y del aparato respiratorio.

El $30 \%$ restante reportan no tener ninguna enfermedad, lo que confirma que las enfermedades más frecuentes en los ancianos son las degenerativas, las tumorales, las infecciosas, las autoinmunitarias, las disregulativas, las traumáticas y las iatrogénicas (García, s.f.).

En la población adulto mayor observada se encontró que el proceso de envejecimiento por el cual están pasando es propio para la edad, como lo explica la teoría del deterioro orgánico (wear and tear) que hace referencia a que los sistemas se van desgastando y deteriorando por su utilización a lo largo de los años ((Castanedo, s.f.). 
Estos cambios se vieron reflejados en su piel, pues en esta se nota la disminución de su grasa, se ve descolorida, delgada, arrugada, seca y frágil, además de sus cambios corporales como lo es la disminución de la estatura y desgaste de masa muscular, también se observó la pérdida en algunos de ellos de su agudeza auditiva, de su olfato y en la mayoría sus ojos tienden a hundirse por disminución de la grasa orbitaria, es evidente el arco senil, de coloración grisáceo ocasionada por la acumulación de lípidos, por otra parte debido a la pérdida de elasticidad del cristalino se produce la «presbicia» o reducción de la capacidad para enfocar objetos cercanos.

Como se mencionó anteriormente, sus cambios físicos notorios son propios del ciclo de vida por el cual están pasando en estos momentos, aunque es evidente que hay otras patologías que los afectan de vez en cuando, como la gripa, que durante el ejercicio de aprendizaje estuvo presente en varios de los adultos mayores, pues se debe al mismo desgaste de sus cuerpos y de los diferentes sistemas que lo conforman, como se expone en la teoría inmunobiológica que dice que hay una disminución de la capacidad de las células de defensa para producir anticuerpos, por consiguiente hay disminución de la respuesta inmune de los organismos (Castanedo, s.f.).

Durante la investigación se observó que son personas muy activas que coordinan por sí solas sus movimientos corporales y su motricidad.

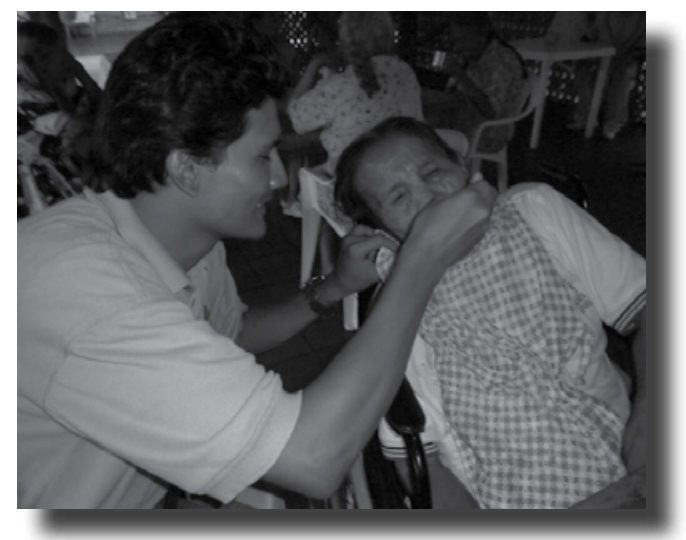

Figura 1: Dimensión Biológica. En esta imagen se presenta a un adulto mayor de género femenino consumiendo sus alimentos, donde se evidencia la dificultad propia para la edad.

La dimensión psicológica presenta las siguientes generalidades: se evidencia un estado de la memoria a largo plazo poco deteriorado, y más específicamente en el caso de las memorias procedimental y episódica, se presenta un déficit cognitivo propio a la edad en esta población, evidenciado en el deterioro percibido en la memoria a mediano plazo, con base en las pruebas MMSE modificado y a la escala de Pfeffer. 
Además se afirma que es propio de la edad según los doctores Pedro Casanova Sotolongo, Pedro Casanova Carrillo y el Liccenciado Carlos Casanova Carrillo de Cuba. El Deterioro de la Memoria Asociado con la Edad (DEMAE) es un trastorno relativamente benigno que puede ser bastante común por el hecho de estar vinculado con el envejecimiento normal y el evidente incremento de la expectativa de vida en el ámbito mundial y nacional, con una mayor población de personas de la tercera edad y el consecuente aumento de enfermedades crónicas, propias de este segmento poblacional. En particular, las demencias y los trastornos cognitivos se han convertido en un problema importante de salud, por su frecuencia en la práctica médica comunitaria cubana y por su transcendencia en la calidad de vida de los enfermos (Casanova et. al., 2004).

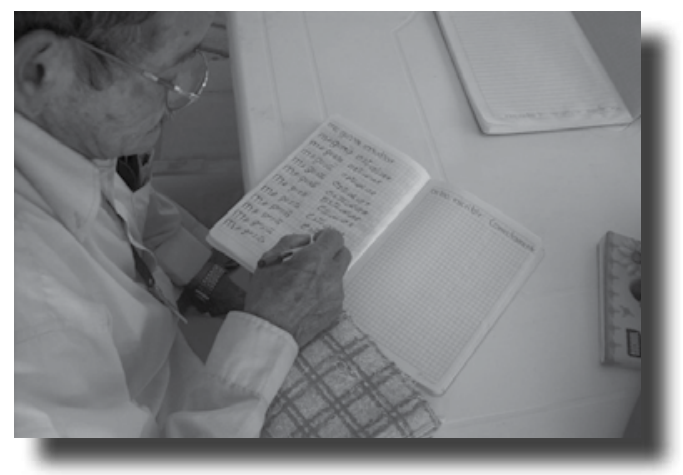

Figura 2. En esta imagen se presenta a un adulto mayor de género masculino, se evidencia un buen estado de ánimo.

En cuanto al aspecto emocional, los adultos mayores muestran estabilidad o equilibrio general, sobresale su sentido del humor, poseen una tranquilidad característica, tienden a ser amables, agradecidos con lo que se les brinda, su estado de ánimo es adecuado, mantienen su sistema de creencias religiosas como parte de su motivación y con base en la valoración emocional de la escala geriátrica de depresión de Yesavage, estos sujetos presentan un estado emocional adecuado a su edad.

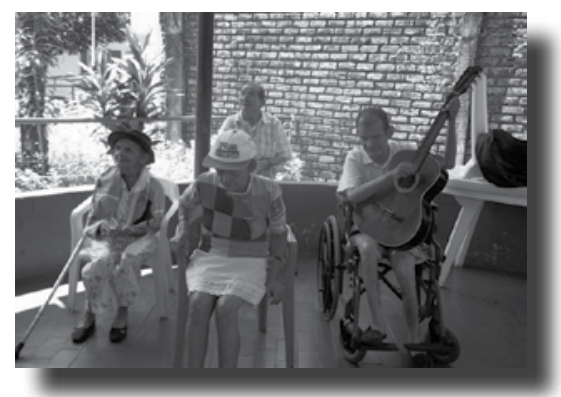

Figura 3. En esta imagen se presenta a un adulto mayor de género femenino compartiendo sus experiencias de vida. 
En cuanto al factor contextual, la mayoría de ellos viven con familiares, lo que constituye una mayor garantía para su bienestar físico y espiritual. Con relación al estado civil de los adultos mayores que asisten a los comedores se observó que la mayoría conviven aun con su pareja, factor que les ayuda a mantener su estabilidad emocional, además muchos de ellos aún conservan sus trabajos y quienes no, a pesar de su edad, buscan la forma de ser productivos para sus hogares ya que como se menciona anteriormente son adultos muy activos e inquietos.

\section{Conclusiones}

El adulto mayor requiere de la inclusión en distintas actividades que le posibilite acceder a una ocupación y que favorezca el desarrollo psicosocial y permita la potencialización de una vida emocional y productiva al máximo.

El envejecer no implica para los seres humanos, estar exentos de posibilidades de ocupar posiciones que los favorezcan en el buen desarrollo emocional, social, y psicosocial, la sociedad cada día debe buscar el incremento de esfuerzos encaminados al bienestar y calidad de vida de este grupo de personas y se constituye en un reto para los profesionales y la sociedad, el contribuir a que esta etapa de la vida sea cada vez más satisfactoria.

Dentro del contexto psicológico, la intervención psicosocial en esta población de adultos mayores debe tener en cuenta el déficit cognitivo que presentan y se recomienda realizar ejercicios o talleres para estimular el aspecto cognitivo, tales como el uso de estrategias mnemotécnicas, ejercicios de atención selectiva, ejercicios de agrupación o categorización, ejercicios de pares asociados de palabras, ejercicios de recuerdo de rostros y lugares, ejercicios de referencias espaciales, así como de referencias temporales.

\section{Referencias bibliográficas}

Casanova S., Pedro, Pedro Casanova C. y Carlos Casanova C. 2004. «Deterioro cognitivo en la tercera edad». en Revista cubana de medicina general integral. 20 (5-6). (Extraído de internet el 11 de julio de 2011 en http://bvs.sld.cu/revistas/mgi/vol20_56_04/mgi125_604.htm).

Castanedo, Cristina. s.f. "Consideraciones generales sobre el envejecimiento». (Extraído de internet el 10 de julio de 2011 en http://www.arrakis.es/ seegg/ documentos/libros/pdflibro/Cap1.pdf). 


\section{Revista de}

investigaciones UNAD

Volumen 10. Número 2. Diciembre 2011

García, Mikel. s.f. «Envejecimiento y calidad de vida» en Casa Pía. http://www.casapia. com/informaciones/Envejecimiento-Calidad-De-Vida/Geriatria-En-El-Siglo-XXI.htm. (Extraído de internet el 10 de julio de 2011).

Monsalve, Martha. 2003. "Los adultos mayores en Colombia». en Tiempo. El portal de la psicogerontología. (Extraído de internet el 18 de junio de 2011 en http://www. psicogerontologia.com/monografias/monsalve.htm).

Restrepo, Carlos Mario. 2005 módulo de Psicopatología del adulto y el viejo, Universidad Nacional Abierta y a Distancia -UNAD-. 